\title{
Advancing COVID-19 differentiation with a robust preprocessing and integration of multi-institutional open-repository computer tomography datasets for deep learning analysis
}

\author{
ELEFTHERIOS TRIVIZAKIS ${ }^{1,2}$, NIKOS TSIKNAKIS ${ }^{1}$, EVANGELIA E. VASSALOU ${ }^{3,4}$, \\ GEORGIOS Z. PAPADAKIS ${ }^{1,2}$, DEMETRIOS A. SPANDIDOS ${ }^{5}$, DIMOSTHENIS SARIGIANNIS ${ }^{6,7}$, \\ ARISTIDIS TSATSAKIS ${ }^{8}$, NIKOLAOS PAPANIKOLAOU ${ }^{1,9}$, \\ APOSTOLOS H. KARANTANAS ${ }^{1-3}$ and KOSTAS MARIAS ${ }^{1,10}$
}

${ }^{1}$ Computational Biomedicine Laboratory (CBML), Foundation for Research and Technology Hellas (FORTH), 70013 Heraklion; ${ }^{2}$ Department of Radiology, Medical School, University of Crete, 71003 Heraklion;

${ }^{3}$ Department of Medical Imaging, University Hospital of Heraklion, 71110 Heraklion; ${ }^{4}$ Department of Radiology,

Sitia District Hospital, 72300 Lasithi; ${ }^{5}$ Laboratory of Clinical Virology, Medical School, University of Crete,

71003 Heraklion; ${ }^{6}$ HERACLES Research Center on the Exposome and Health, Centre for Interdisciplinary Research and Innovation, Aristotle University of Thessaloniki, 57001 Thermi, Greece; ${ }^{7}$ University School for Advanced Studies IUSS, I-27100 Pavia, Italy; ${ }^{8}$ Department of Forensic Sciences and Toxicology, Medical School, University of Crete, 71003 Heraklion; ${ }^{9}$ Computational Clinical Imaging Group, Centre for the Unknown, Champalimaud Foundation, 1400-038 Lisbon, Portugal; ${ }^{10}$ Department of Electrical and Computer Engineering, Hellenic Mediterranean University, 71410 Heraklion, Greece

Received August 14, 2020; Accepted September 11, 2020

DOI: $10.3892 /$ etm.2020.9210

\begin{abstract}
The coronavirus pandemic and its unprecedented consequences globally has spurred the interest of the artificial intelligence research community. A plethora of published studies have investigated the role of imaging such as chest $\mathrm{X}$-rays and computer tomography in coronavirus disease 2019 (COVID-19) automated diagnosis. Open repositories of medical imaging data can play a significant role by promoting cooperation among institutes in a world-wide scale. However, they may induce limitations related to variable data quality and intrinsic differences due to the wide variety of scanner vendors and imaging parameters. In this study, a state-of-the-art custom U-Net model is presented with a dice similarity coefficient performance of $99.6 \%$ along with a transfer learning VGG-19 based model for COVID-19 versus pneumonia differentiation exhibiting an area under curve of $96.1 \%$. The above was significantly improved over the baseline model trained with no segmentation in selected tomographic slices of the
\end{abstract}

Correspondence to: Dr Kostas Marias, Computational Biomedicine Laboratory (CBML), Foundation for Research and Technology Hellas (FORTH), 100 N. Plastira Street, Vassilika Vouton, 70013 Heraklion, Greece

E-mail: kmarias@ics.forth.gr

Key words: artificial intelligence, deep learning analysis, COVID-19, multi-institutional data same dataset. The presented study highlights the importance of a robust preprocessing protocol for image analysis within a heterogeneous imaging dataset and assesses the potential diagnostic value of the presented COVID-19 model by comparing its performance to the state of the art.

\section{Introduction}

Coronavirus disease 2019 (COVID-19) is an infectious disease, caused by the new coronavirus which was first observed in Wuhan, China in December 2019. On the 11th of March 2020, the World Health Organization (WHO) declared this outbreak as a pandemic. As of the 8th of July 2020, more than 11.5 million people have been confirmed to have contracted the virus and more than half a million have died due to complications of the disease (1). The clinical symptoms of COVID-19 are non-specific and in most of the cases include fever, cough, fatigue and dyspnea (2). Obesity (3), chronic cardiovascular diseases (4) and smoking habits (5) have also been reported to contribute to the deterioration of the disease. Toxic stressors in urban environments could have played a role in deteriorating the immune system of the local population forming the basis for the spreading of COVID-19 disease (6). In a similar context, Tsatsakis et al (7) explored the association among human-induced pollutants found in greenhouse gases, the effect on the immune system and other environmental aspects related to the COVID-19 pandemic. Early diagnosis is important not only for prompt treatment planning but also for isolation of patients in order to prevent spreading the virus to the community. Currently, reverse transcription-polymerase 
chain reaction (RT-PCR) represents the gold-standard for diagnosing COVID-19. However, testing with RT-PCR shows limited sensitivity ( $\mathrm{SN}$ ) which, adding to the shortage of testing kits and the increased waiting time for results, increase the screening burden and delays the isolation procedure $(8,9)$. Thus, the scientific community has been searching alternative protocols for timely and accurate diagnosis. X-ray and chest computed tomography (CT) imaging could be used as a reliable and rapid approach for COVID-19 screening $(9,10)$. Both methods have the potential to depict COVID-19 related chest abnormalities, with ground-glass lung opacification and consolidation showing bilateral and subpleural or diffuse distribution representing the cardinal findings (9,11-16). Although, the image acquisition is easy and fast, interpretation can be challenging and time-consuming, especially for inexperienced and subspecialized medical professionals. In order to eliminate such drawbacks, the scientific community has been shifting its focus towards developing automated tools for the analysis of imaging data. In this context, several artificial intelligence (AI) methods have been developed to provide a prediction for the disease and preliminary severity assessment. Tsiknakis et al (17) proposed an Interpretable Convolutional Neural Network (CNN) based on transfer learning for predicting COVID-19 against viral and bacterial pneumonia and normal cases based on more than 400 X-ray images, achieving an area under curve (AUC) of $100 \%$. Apostolopoulos and Mpesiana (18) also developed a CNN for predicting COVID-19 from almost 1,500 X-ray images, achieving an accuracy (ACC) of $96.78 \%, \mathrm{SN}$ of $98.66 \%$ and specificity (SPC) of $96.46 \%$. However, compared to X-ray images, CT scans provide a more detailed overview of the internal structure of lung parenchyma due to the lack of overlapping tissues. Thus, recent research has focused on the development of effective AI methods based on CT scans. Li et al (19) developed a 3D CNN for predicting COVID-19 against community acquired pneumonia (CAP) from 3D CT scans. Their model was trained on a fairly large dataset of 4,356 CT scans from 3,322 patients, 1,296 of which referred to COVID-19 positive patients, and achieved SN of 90\%, SPC of $96 \%$ and an AUC equal to $95 \%$ regarding the COVID-19 class. Their proposed model consists of several identical ResNet50 models, one for each CT image. The feature maps of all the backbone models were combined through a max pooling layer, which was followed by a dense layer for the final ternary classification. The main downside of such an approach is that only one ground truth label per exam is available, and not one for each layer of the CT scan. Optimally, for achieving a finer and detailed network performance, each CT layer should be graded individually. In this way, the model would benefit from the inter-CT-layer relations of the $3 \mathrm{D}$ input, as well as from the extended ground truth information. Wang et al (20) proposed a CNN model which was first trained on CT scans with cancer from 4,106 patients in order to learn lung features. Subsequently, they fine-tuned the model on a COVID-19 multi-centric CT dataset (709 patients). Their model was validated on 4 independent datasets from different clinical centers. Their approach performed well in identifying COVID-19 against bacterial or other viral pneumonia cases, achieving an AUC of $87 \%$, SN of $80.39 \%$ and SPC of $76.61 \%$ against bacterial pneumonia and AUC of $86 \%$, SN of $79.35 \%$ and SPC of $71.43 \%$ against viral pneumonia. In addition, they stratified the patients into high and low risk groups, in order to propose a hospital-stay time schedule. Zhang et al (21) utilized another network to segment the lung regions, before applying the classification network in order to discard the background and irrelevant regions within the $\mathrm{CT}$ scan. Their proposed pipeline achieved an ACC of $91.2 \%$, SN of $94.03 \%$, SPC of $88.46 \%$ and an AUC of $96.1 \%$ for predicting COVID-19 on a prospective Chinese dataset, while similar results were achieved on two other prospective Chinese datasets. Subsequently, the model was applied on a dataset from Ecuador, ACC of $84.11 \%, \mathrm{SN}$ of $86.67 \%$, SPC of $82.26 \%$ and AUC of $90.5 \%$. In addition, they evaluated the effect of drug treatment on lesion size and volume changes using their AI model. Finally, they compared their model to 8 junior and 4 mid-senior radiologists, for predicting COVID-19 on an independent dataset which was annotated by 4 independent senior radiologists. The model outperformed the junior radiologists, while its performance was comparable to that of the mid-senior ones. Ardakani et al (22) utilized 10 pre-trained convolutional networks as the backbone of their classifier, achieving the best AUC score of $99.4 \%, \mathrm{SN}$ of $100 \%$, SPC of $99 \%$ and ACC of $99.5 \%$, for distinguishing COVID-19 pneumonia from other types of pneumonia (viral and bacterial). At the same time, the performance of a radiologist was moderate, achieving much lower results, i.e., AUC of $87.3 \%$, SN of $89 \%$, SPC of $83 \%$ and ACC of $86 \%$. However, their dataset included a limited amount of data, namely $1,020 \mathrm{CT}$ slices for 108 COVID-19 positive patients and 86 pneumonia positive patients. Additionally, plenty of unpublished scientific preprints have been available on open databases, claiming high accuracy, SN and SPC scores (23-29), for predicting COVID-19 against other pneumonia types or healthy patients based on deep learning models.

In this study, we propose an extensive pipeline for automatic COVID-19 screening against other types of pneumonia (i.e., viral and bacterial) from CT scans, utilizing lung segmentation for increasing the accuracy. Some key innovations of this study can be summarized in the integration of multi-institutional and open-access data from a variety of scanners and imaging protocols retrieved from online repositories in formats such as DICOM or Portable network Graphics (png), the development of a deep learning lung segmentation model for multiple CT window settings and finally a deep learning model for differentiating COVID-19 from CAP. This study introduces a state-of-the-art deep learning model for lung segmentation on slices with a variety of CT window settings (DSC 99.6\%) and an image analysis deep model trained with multi-institutional data for differentiating COVID-19 from CAP (AUC 96.1\%).

\section{Materials and methods}

Dataset. The proposed analysis was performed using open data of confirmed COVID-19 and widely available online cases. In particular, the patient cohort consisted of 3 datasets (30-32) with 1,266 COVID-19 and 586 pneumonia cases stratified on a patient-basis resulting in 5,109 CT slices. Furthermore, slices characterized other than COVID-19 or pneumonia such as normal were discarded. The curated individual slices were collected from patients in hospitals from São Paulo in Brazil (30) and open repositories such as medRxiv 


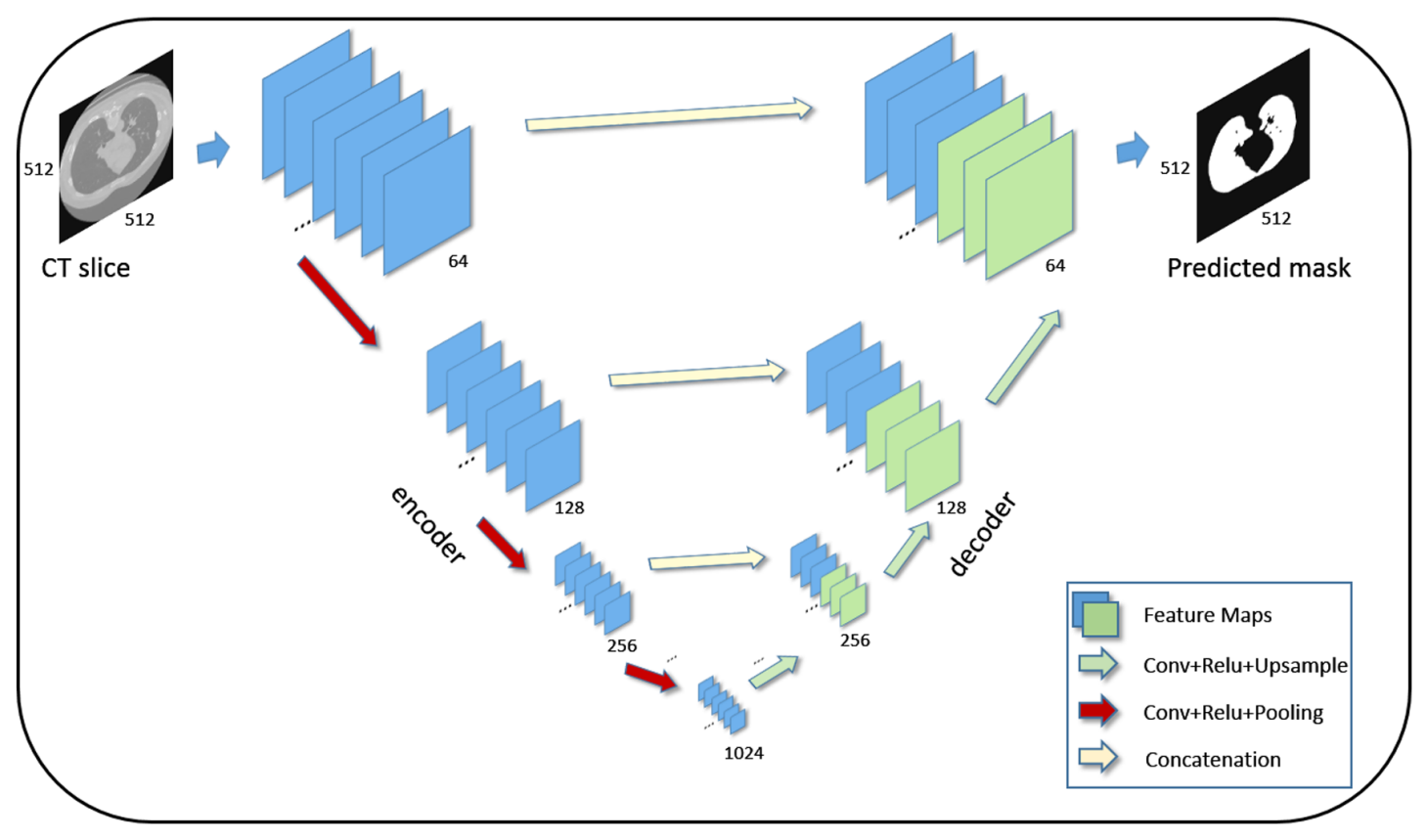

Figure 1. An overview of the used custom U-Net segmentation architecture.

(https://www.medrxiv.org/) and bioRxiv (https://www.biorxiv. org/) (31). Additionally, these datasets were made available as stand alone 8-bit image files with unknown compression methodology, no metadata information available and various CT window settings rendering segmentation and classification tasks with traditional methods challenging. In contrast, the dataset (32) consisted of 20 cases with COVID-19 pixel-based annotated regions of interest in meta-image form (NifTi files) from the open repositories of Radiopedia (http://radiopedia.org) and Coronacases (http://coronacases.org). This multi-institutional collection of CT examinations lead to high variability in spacing, pixel array size and windowing type across the examined cases.

Lung segmentation. U-Net (33) is a fully-convolutional architecture comprising multiple consecutive convolutional, pooling layers in the encoder part and convolutional, upsampling layers in the decoder part. The deep network can accurately match incoming CT examination slices to their corresponding segmentation mask. This process is learned from data with known segmentation masks through the back-propagation of the similarity error during the training phase. The Lung Image Database Consortium-Image Database Resource Initiative (LIDC-IDRI) (34) dataset was used for training and evaluating the deep learning segmentation model. The ground truth masks for lung segmentation were extracted by a fully-automated Hounsfield Units (HU) based algorithm (35). A subset of the 1,018 scans with 98,433 CT slices was used for model convergence. A detailed view of the architecture is depicted in (Fig. 1).

Pre-processing. The image resolution of the examined patient cohort varied from $148 \times 61$ to $1,637 \times 1,225$ pixels introducing a significant limitation for the deep learning analysis. Each slice was resized with a linear interpolation technique targeting $512 \times 512$ pixels to match the required dimension of input layer of the U-Net segmentation network. The slices of the examined cohort were segmented with the custom U-Net and normalized to achieve pixel values with unit variance and zero mean prior to the deep learning analysis. In Fig. 2 the testing set ground truth masks versus predicted masks are presented revealing a highly performing segmentation model and in Fig. 3 a randomly selected set of predicted segmentation masks from the COVID-19 dataset are demonstrated. Despite the aforementioned variability in pixel array dimensions and CT window settings the proposed methodology establishes minimal segmentation error.

Deep architecture analysis. A transfer learning approach was used for improving the convergence and fine-tuning process based on CT slices of the examined cohort by adapting the inner representation of the pre-trained model to the targeted COVID-19 versus CAP analysis. Two additional convolutional layers with 256 filters each and a neural network with 1,000 neurons were appended in the original pre-trained model as depicted in Fig. 4. The source code and the detailed hyperparameter configuration files are provided online (https:/github.com/trivizakis/ct-covid-analysis).

Performance evaluation. The evaluation was assessed by following metrics of $\mathrm{ACC}, \mathrm{SN}, \mathrm{SPC}$ and precision (PR):

$$
\begin{aligned}
& A C C=\frac{T P+T N}{T P+F P+T N+F N} \\
& S N=\frac{T P}{T P+F N} \\
& S P C=\frac{T N}{T N+F P} \\
& P R=\frac{T P}{T P+F P}
\end{aligned}
$$



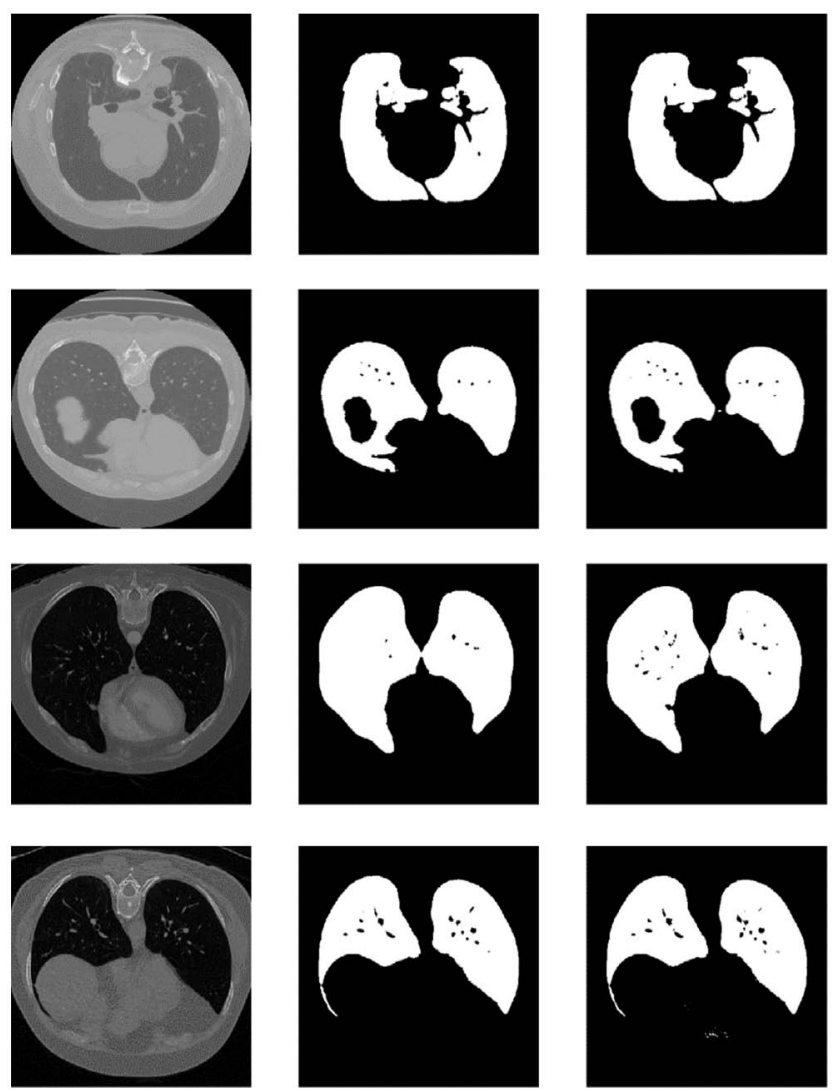

Figure 2. Testing samples of the LIDC-IDRI: original CT slices (left), ground truth (center) and U-Net predicted segmentation mask (right). LIDC-IDRI, Lung Image Database Consortium-Image Database Resource Initiative; CT, computed tomography.
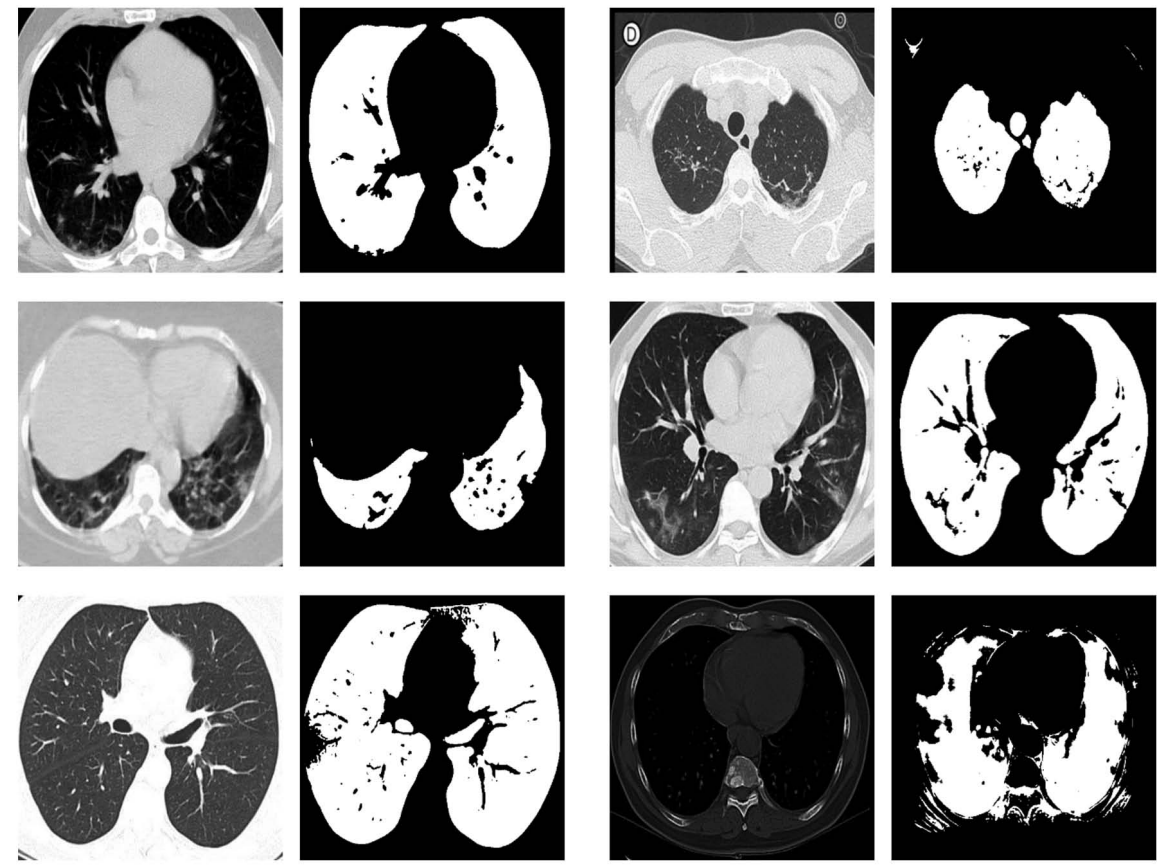

Figure 3. Sample slices of the examined analysis cohort with lung segmentation masks predicted by U-Net.

\section{Results}

A 4-fold cross-validation process was performed for splitting the dataset in the convergence and the testing set. The convergence set was further randomly split into the training $(80 \%)$ and validation set (20\%) for applying the fine-tuning and hyperparameter optimization process respectively. The testing folds remained unseen throughout the analysis to assess the performance of the proposed deep learning model. A custom U-Net for lung parenchyma segmentation was trained and 

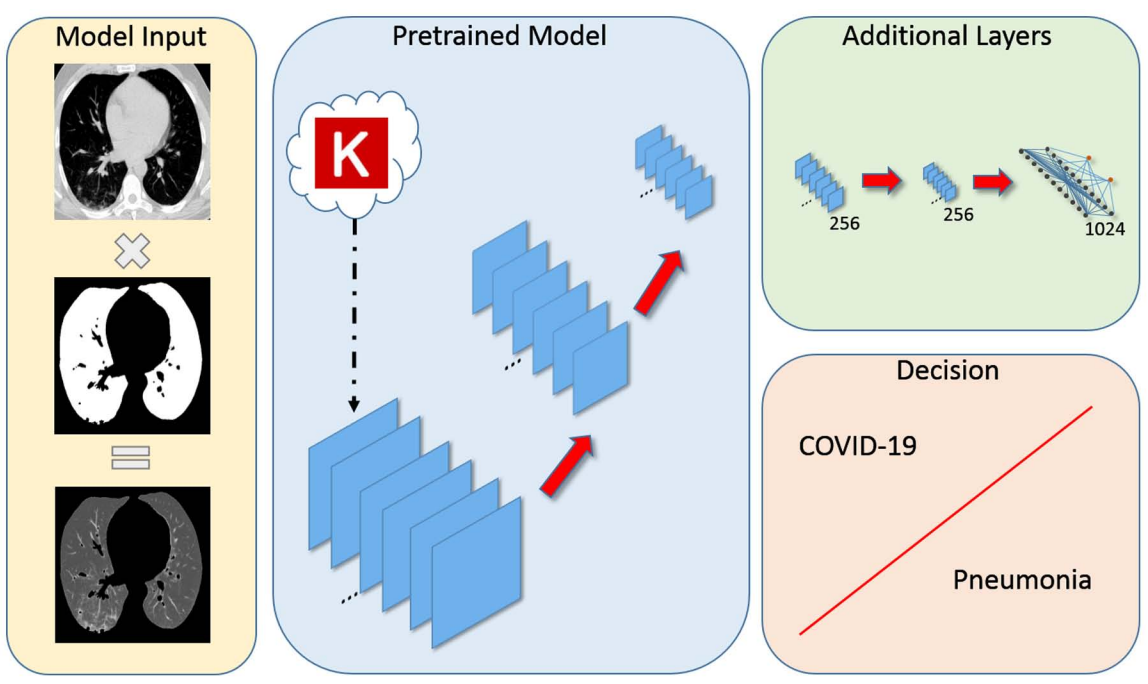

Figure 4. The proposed transfer learning analysis pipeline for COVID-19 versus pneumonia differentiation including the preprocessing phase with lung segmentation and z-normalization.
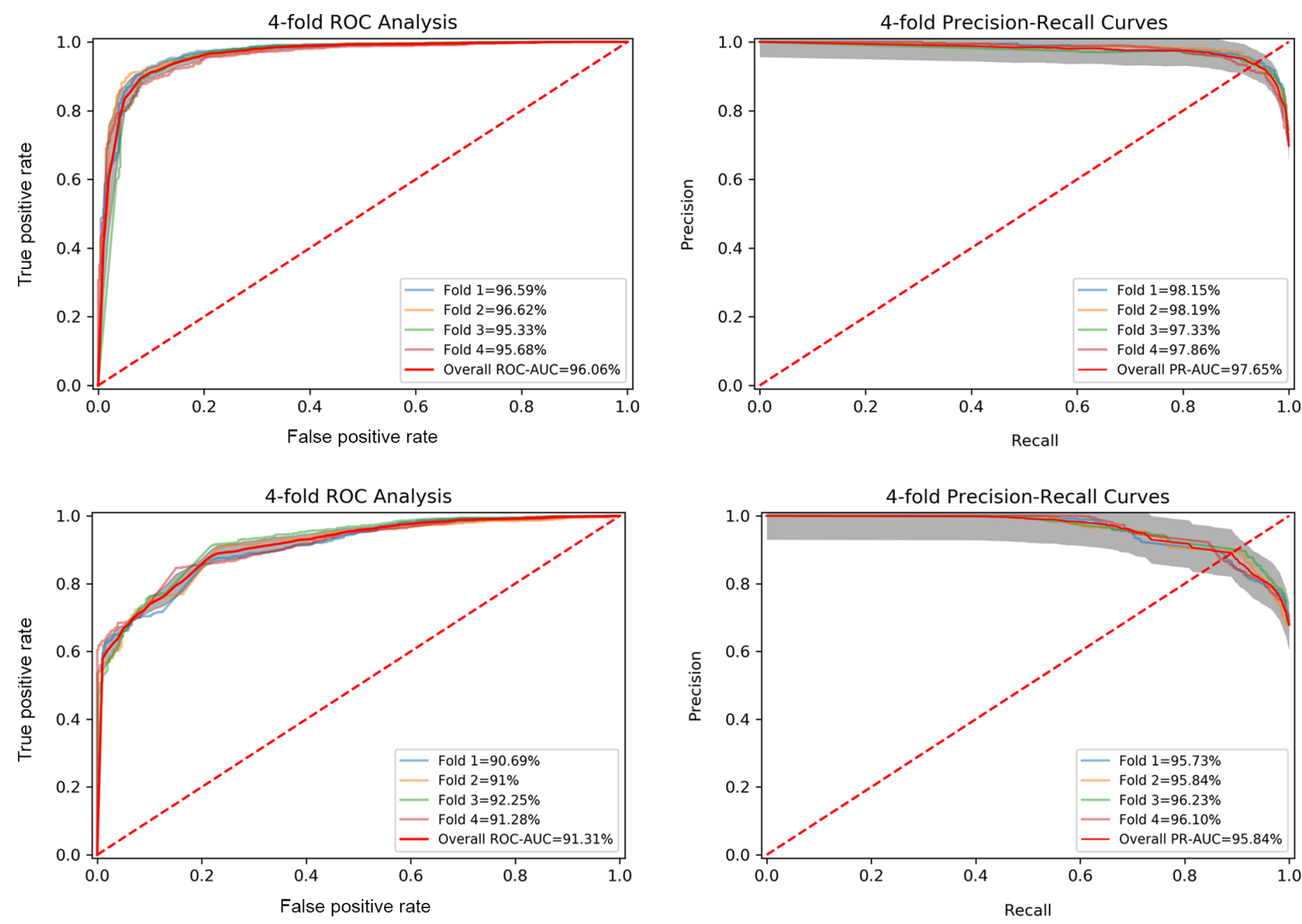

Figure 5. Performance assessment with ROC (left), Precision-Recall (right) curves for the proposed analysis (top) and the baseline model (bottom). ROC, receiver operating characteristic.

evaluated on a total set of 109,370 LIDC-IDRI CT slices with ground truth segmentation masks calculated on a HU basis by an automated algorithm. The U-Net converged after 5 epochs of training and achieved a performance of Dice Similarity Coefficient of $99.55 \%$ in the testing set providing a reliable and accurate segmentation model as evident by the randomly selected examples presented in Fig. 3. Although, perfect segmentation masks cannot be obtained for the analysis dataset due to the variability in image quality; the majority of pathological pixels are present in the final segmented image despite some false negative pixels. Several pre-trained models were examined including VGG (36), Inception (37), NASNet (38), DenseNet (39) and MobileNet (40) with additional convolutional and neural network layers as depicted in 
Table I. Performance of the proposed analysis in comparison to the current peer-reviewed research with similar end-points.

\begin{tabular}{|c|c|c|c|c|c|c|}
\hline Type \% & $\mathrm{ACC}$ & $\mathrm{SN}$ & SPC & AUC & $\begin{array}{l}\text { Patients, no. } \\
\text { (training/testing) }\end{array}$ & $\begin{array}{l}\text { CT Slices, no. } \\
\text { (training/testing) }\end{array}$ \\
\hline Proposed with segmentation & 91.1 & 92.0 & 87.5 & 96.1 & $\begin{array}{l}\text { COVID-19: 894/372 } \\
\text { CAP: } 414 / 172\end{array}$ & $\begin{array}{l}\text { COVID-19: 2,047/853 } \\
\text { CAP: 976/406 }\end{array}$ \\
\hline Proposed no lung segmentation & 85.1 & 88.8 & 76.8 & 91.3 & & \\
\hline Li et al (19) & - & 90.0 & 96.0 & 95.0 & $\begin{array}{l}\text { COVID-19: 400/68 } \\
\text { CAP: 1396/155 } \\
\text { Non-pneumonia: } 1,173 / 130\end{array}$ & $\begin{array}{l}\text { COVID-19: } 1,165 / 127 \\
\text { CAP: } 1,560 / 175 \\
\text { Non-pneumonia: } 1,193 / 132\end{array}$ \\
\hline Wang et al (20) & - & 80.4 & 76.6 & 87.0 & All: $709 / 557$ & - \\
\hline Zhang et al (21) & 91.2 & 94.0 & 88.5 & 96.0 & $\begin{array}{l}\text { COVID-19: } 752 \\
\text { CAP: } 797 \\
\text { Non-pneumonia: } 697\end{array}$ & All: 444.034 \\
\hline Ardakani et al (22) & 99.5 & 100.0 & 99.0 & 99.4 & $\begin{array}{l}\text { COVID-19: } 108 \\
\text { Non-COVID-19: } 86\end{array}$ & $\begin{array}{l}\text { COVID-19: } 510 \\
\text { Non-COVID-19: } 510 \\
\text { Training/testing (All): 816/102 }\end{array}$ \\
\hline Harmon et al (42) & 90.8 & 84.0 & 93.0 & 94.8 & $\begin{array}{l}\text { COVID-19: 451/276 } \\
\text { Non-COVID-19: 533/1,011 }\end{array}$ & 3D classification model \\
\hline
\end{tabular}

ACC, achieving an accuracy; SN, sensitivity; SPC, specificity; AUC, area under curve; CT, computed tomography; CAP, community acquired pneumonia; COVID-19, coronavirus disease 2019.

Fig. 4. The analysis architecture was trained on average for 16 epochs before early-stopping. Most of pre-trained models preformed comparably with an AUC up to $93 \%$ and the highest performance (AUC 96.1\%) was achieved with VGG-19 as the backend pre-trained network. The receiver operating characteristic (ROC) and PR-recall curves of the best model are presented in Fig. 5 (top) outlining the robustness and constancy of the examined analysis. The proposed deep learning architecture with a robust pre-processing protocol including lung segmentation and image standardization outperforms the baseline model (up-to AUC 91.3\%) with similar architecture but with unsegmented CT slices and the current literature (Table I) with similar experimental protocols.

\section{Discussion}

The proposed analysis integrates deep learning lung segmentation for removing irrelevant organs/landmarks and transfer learning for discriminating between COVID-19 and other types of pneumonia from CT imaging data with multiple window settings. The segmentation model was trained on openly available CT data (LIDC-IDRI) providing state-of-the-art performance even in datasets such as the examined COVID-19 (Fig. 3) from various institutes with different CT scanners, no DICOM metadata available and variability in image quality. Thus, the transfer learning-based analysis was focused only on the lung region resulting in higher performance (AUC 96.1\%) significantly advancing the baseline of $91.3 \%$ without segmented CT slices of the examined dataset. The fact that during inference, suspicious or disputable CT slices selected for analysis by the deep model should have been identified by an expert radiologist from the raw $\mathrm{CT}$ volume, might be considered as a limitation since a fully automated diagnosis is not possible. That said, as presented in Table I, the proposed methodology outperforms the current literature (19-21) in terms of AUC performance. In particular, the deep model proposed by Zhang et al (21) demonstrates similar performance with the present analysis but the examined CT slices were manually segmented on lesion basis before classification (COVID-19 versus common pneumonia versus normal patients). Ardakani et al (22) claims a model with an AUC performance of $99.4 \%$, however, it utilizes a different experimental protocol than the examined herein, in which patches (60 by 60 pixels) were extracted by an experienced radiologist for the analysis requiring additional and time-consuming labor from a clinical practice perspective.

In conclusion, the present study is addressing limitations of other efforts $(22,27,41)$ where the analysis was applied on raw $\mathrm{CT}$ data with more demanding preprocessing phase and uniform data quality. This is further highlighted by the increased performance stability demonstrated by the low prediction variability among deep models of each fold in both ROC (standard deviation $0.55 \%$ ) and PR (standard deviation $0.34 \%$ ) curves as depicted in Fig. 5 (top).

\section{Acknowledgements}

Not applicable.

\section{Funding}

Part of this study was financially supported by the Stavros Niarchos Foundation within the framework of the project ARCHERS ('Advancing Young Researchers' Human Capital 
in Cutting Edge Technologies in the Preservation of Cultural Heritage and the Tackling of Societal Challenges').

\section{Availability of data and materials}

Not applicable.

\section{Authors' contributions}

ET, NT and KM conceived and designed the study. ET, NT and $\mathrm{KM}$ researched the literature, performed analysis and interpretation of data and drafted the manuscript. EEV, GZP, AHK, NP, DAS, DS and AT critically revised the article for important intellectual content and assisted in the literature search for this article. All authors agree to be accountable for all aspects of the work in ensuring that questions related to the accuracy or integrity of any part of the work are appropriately investigated, and finally approved the version of the manuscript to be published.

\section{Ethics approval and consent to participate}

Not applicable.

\section{Patient consent for publication}

Not applicable.

\section{Competing interests}

DAS is the Editor-in-Chief for the journal, but had no personal involvement in the reviewing process, or any influence in terms of adjudicating on the final decision, for this article. All the other authors declare that they have no competing interests.

\section{References}

1. World Health Organization (WHO): WHO Coronavirus Disease (COVID-19) Dashboard. WHO, Geneva, 2020. https://covid19. who.int/. Accessed August 1, 2020.

2. World Health Organization (WHO): Report of the WHO-China Joint Mission on Coronavirus Disease 2019 (COVID-19). WHO, Geneva, 2020. https://www.who.int/docs/default-source/coronaviruse/who-china-joint-mission-on-covid-19-final-report.pdf. Accessed February 28, 2020.

3. Petrakis D, Margină D, Tsarouhas K, Tekos F, Stan M, Nikitovic D, Kouretas D, Spandidos DA and Tsatsakis A: Obesity a risk factor for increased COVID-19 prevalence, severity and lethality (Review). Mol Med Rep 22: 9-19, 2020.

4. Docea AO, Tsatsakis A, Albulescu D, Cristea O, Zlatian O, Vinceti M, Moschos SA, Tsoukalas D, Goumenou M, Drakoulis N, et al: A new threat from an old enemy: Re-emergence of coronavirus (Review). Int J Mol Med 45: 1631-1643, 2020.

5. Farsalinos K, Niaura R, Le Houezec J, Barbouni A, Tsatsakis A, Kouretas D, Vantarakis A and Poulas K: Editorial: Nicotine and SARS-CoV-2: COVID-19 may be a disease of the nicotinic cholinergic system. Toxicol Rep 7: 658-663, 2020.

6. Kostoff RN, Briggs MB, Porter AL, Hernández AF, Abdollahi M, Aschner $\mathrm{M}$ and Tsatsakis A: The under-reported role of toxic substance exposures in the COVID-19 pandemic. Food Chem Toxicol: Aug 14, 2020 (Epub ahead of print).

7. Tsatsakis A, Petrakis D, Nikolouzakis TK, Docea AO, Calina D, Vinceti M, Goumenou M, Kostoff RN, Mamoulakis C, Aschner M, et al: COVID-19, an opportunity to reevaluate the correlation between long-term effects of anthropogenic pollutants on viral epidemic/pandemic events and prevalence. Food Chem Toxicol 141: 111418, 2020.
8. Xie X, Zhong Z, Zhao W, Zheng C, Wang F and Liu J: Chest CT for typical coronavirus disease 2019 (COVID-19) pneumonia: Relationship to negative RT-PCR testing. Radiology 296: E41-E45, 2020.

9. Ai T, Yang Z, Hou H, Zhan C, Chen C, Lv W, Tao Q, Sun Z and Xia L: Correlation of chest CT and RT-PCR testing for coronavirus disease 2019 (COVID-19) in China: A Report of 1014 cases. Radiology 296: E32-E40, 2020.

10. Fang Y, Zhang H, Xie J, Lin M, Ying L, Pang P and Ji W: Sensitivity of chest CT for COVID-19: comparison to RT-PCR. Radiology 296: E115-E117, 2020.

11. Chen N, Zhou M, Dong X, Qu J, Gong F, Han Y, Qiu Y, Wang J, Liu Y, Wei Y, et al: Epidemiological and clinical characteristics of 99 cases of 2019 novel coronavirus pneumonia in Wuhan, China: A descriptive study. Lancet 395: 507-513, 2020.

12. Holshue ML, DeBolt C, Lindquist S, Lofy KH, Wiesman J, Bruce H, Spitters C, Ericson K, Wilkerson S, Tural A, et al; Washington State 2019-nCoV Case Investigation Team: First case of 2019 novel coronavirus in the United States. N Engl J Med 382: 929-936, 2020.

13. Wang D, Hu B, Hu C, Zhu F, Liu X, Zhang J, Wang B, Xiang H, Cheng Z, Xiong Y, et al: Clinical characteristics of 138 hospitalized patients with 2019 novel coronavirus-infected pneumonia in Wuhan, China. JAMA 323: 1061-1069, 2020.

14. Li Q, Guan X, Wu P, Wang X, Zhou L, Tong Y, Ren R, Leung KSM, Lau EHY, Wong JY, et al: Early transmission dynamics in Wuhan, China, of novel coronavirus-infected pneumonia. N Engl J Med 382: 1199-1207, 2020.

15. Chung M, Bernheim A, Mei X, Zhang N, Huang M, Zeng X, Cui J, Xu W, Yang Y, Fayad ZA, et al: CT imaging features of 2019 novel coronavirus (2019-NCoV). Radiology 295: 202-207, 2020.

16. Huang C, Wang Y, Li X, Ren L, Zhao J, Hu Y, Zhang L, Fan G, Xu J, Gu X, et al: Clinical features of patients infected with 2019 novel coronavirus in Wuhan, China. Lancet 395: 497-506, 2020.

17. Tsiknakis N, Trivizakis E, Vassalou EE, Papadakis GZ, Spandidos DA,Tsatsakis A, Sánchez-García J,López-GonzálezR, Papanikolaou N, Karantanas AH, et al: Interpretable artificial intelligence framework for COVID-19 screening on chest X-rays. Exp Ther Med 20: 727-735, 2020.

18. Apostolopoulos ID and Mpesiana TA: Covid-19: automatic detection from X-ray images utilizing transfer learning with convolutional neural networks. Phys Eng Sci Med 43: 635-640, 2020.

19. Li L, Qin L, Xu Z, Yin Y, Wang X, Kong B, Bai J, Lu Y, Fang Z, Song Q, et al: Using artificial intelligence to detect COVID-19 and community-acquired pneumonia based on pulmonary CT: Evaluation of the diagnostic accuracy. Radiology 296: E65-E71, 2020.

20. Wang S, Zha Y, Li W, Wu Q, Li X, Niu M, Wang M, Qiu X, $\mathrm{Li} \mathrm{H}, \mathrm{Yu} \mathrm{H}$, et al: A fully automatic deep learning system for COVID-19 diagnostic and prognostic analysis. Eur Respir J 56: 2000775, 2020.

21. Zhang K, Liu X, Shen J, Li Z, Sang Y, Wu X, Zha Y, Liang W, Wang C, Wang K, et al: Clinically applicable AI system for accurate diagnosis, quantitative measurements, and prognosis of COVID-19 pneumonia using computed tomography. Cell 181: 1423-1433.e11,2020.

22. Ardakani AA, Kanafi AR, Acharya UR, Khadem N and Mohammadi A: Application of deep learning technique to manage COVID-19 in routine clinical practice using CT images: Results of 10 convolutional neural networks. Comput Biol Med 121: 103795, 2020

23. Song Y, Zheng S, Li L, Zhang X, Zhang X, Huang Z, Chen J, Zhao H, Jie Y, Wang R, et al: Deep learning enables accurate diagnosis of novel coronavirus (COVID-19) with CT images. medRxiv: doi: https://doi.org/10.1101/2020.02.23.20026930.

24. Zheng C, Deng X, Fu Q, Zhou Q, Feng J, Ma H, Liu W and Wang X: Deep learning-based detection for COVID-19 from chest CT using weak label. medRxiv: doi: https://doi.org/10.1101 /2020.03.12.20027185.

25. Gozes O, Frid-Adar M, Greenspan H, Browning PD, Zhang H, Ji W, Bernheim A and Siegel E: Rapid AI development cycle for the coronavirus (COVID-19) pandemic: Initial results for automated detection and patient monitoring using deep learning CT image analysis. arXiv:2003.05037.

26. Shan F, Gao Y, Wang J, Shi W, Shi N, Han M, Xue Z, Shen D and Shi Y: Lung infection quantification of COVID-19 in CT images with deep learning. arXiv:2003.04655. 
27. Kassani SH, Kassasni PH, Wesolowski MJ, Schneider KA and Deters R: Automatic detection of coronavirus disease (COVID-19) in x-ray and CT images: A machine learning-based approach. arXiv:2004.10641.

28. Hu S, Gao Y, Niu Z, Jiang Y, Li L, Xiao X, Wang M, Fang EF, Menpes-Smith W, Xia J, et al: Weakly supervised deep learning for COVID-19 infection detection and classification from CT images. IEEE Access 8: 118869-118883, 2020.

29. Chen X, Yao L and Zhang Y: Residual attention U-Net for automated multi-class segmentation of COVID-19 chest CT images. arXiv:2004.05645.

30. Soares E, Angelov P, Biaso S, Froes MH and Abe DK: SARS-CoV-2 CT-scan dataset: A large dataset of real patients CT scans for SARS-CoV-2 identification. medRxiv: doi: https:/ doi.org/10.1101/2020.04.24.20078584

31. Yang $X, H e X$, Zhao J, Zhang $Y$, Zhang $S$ and $X$ ie $P$ : COVID-CT-Dataset: A CT scan dataset about COVID-19. arXiv:2003.13865.

32. Ma J, Ge C, Wang Y, An X, Gao J, Yu Z, Zhang M, Liu X, Deng X, Cao S, et al: COVID-19 CT lung and infection segmentation dataset. Zenodo: http://doi.org/10.5281/zenodo.3757476.

33. Ronneberger O, Fischer P and Brox T: U-net: Convolutional networks for biomedical image segmentation. In: Lecture Notes in Computer Science (including subseries Lecture Notes in Artificial Intelligence and Lecture Notes in Bioinformatics). Vol 9351. Springer Verlag, pp234-241, 2015.

34. Armato III S, McLennan G, Bidaut L, McNitt-Gray M, Meyer C, Reeves A, Zhao B, Aberle D, Henschke C, Clarke L, et al: The Lung Image Database Consortium (LIDC) and Image Database Resource Initiative (IDRI): a completed reference database of lung nodules on CT scans. Med Phys 38: 915-931, 2011.
35. gitHub: wanwanbeen/ct_lung_segmentation: Robust segmentation of lung and airway in CT scans. https://github.com/ wanwanbeen?tab=repositories. Updated November 29, 2017.

36. Simonyan K and Zisserman A: Very deep convolutional networks for large-scale image recognition. arXiv:1409.1556.

37. Szegedy C, Vanhoucke V, Ioffe S, Shlens J and Wojna Z: Rethinking the inception architecture for computer vision. In: Proceedings of the 2016 IEEE Conference on Computer Vision and Pattern Recognition (CVPR), Las Vegas, NV, pp2818-2826, 2016.

38. Zoph B, Vasudevan V, Shlens J and Le QV: Learning transferable architectures for scalable image recognition. arXiv:1707.07012v4.

39. Huang G, Liu Z, van der Maaten L and Weinberger KQ: Densely connected convolutional networks. arXiv:1608.06993.

40. Sandler M, Howard A, Zhu M, Zhmoginov A and Chen LC: MobileNetV2: Inverted Residuals and Linear Bottlenecks. The IEEE Conference on Computer Vision and Pattern Recognition (CVPR), pp4510-4520, 2018

41. He X, Yang X, Zhang S, Zhao J, Zhang Y, Xing E and Xie P: Sample-Efficient Deep Learning for COVID-19 Diagnosis Based on CT Scans. medRxiv: doi: https://doi.org/10.1101/2020.04.13. 20063941

42. Harmon SA, Sanford TH, Xu S, Turkbey EB, Roth H, Xu Z, Yang D, Myronenko A, Anderson V, Amalou A, et al: Artificial intelligence for the detection of COVID-19 pneumonia on chest CT using multinational datasets. Nat Commun 11: 4080, 2020.

(i) $($ ) This work is licensed under a Creative Commons Attribution-NonCommercial-NoDerivatives 4.0 International (CC BY-NC-ND 4.0) License. 